\title{
Forearm and hand muscles exhibit high coactivation and overlapping of cortical motor representations
}

Gabriela P. Tardelli ${ }^{a \dagger}$, Victor Hugo Souza ${ }^{\mathrm{a}, \mathrm{b}, \mathrm{c} \dagger}$, Renan H. Matsuda ${ }^{\mathrm{a}, \mathrm{b}}$, Marco A. C. Garciaa ${ }^{\mathrm{a}, \mathrm{c}, \mathrm{d}}$, Pavel A. Novikove, Maria A. Nazarova ${ }^{\mathrm{e}, \mathrm{f}}$, Oswaldo Baffa ${ }^{\mathrm{a}}$

a Department of Physics, School of Philosophy, Science and Literature of Ribeirão Preto, University of São Paulo, Ribeirão Preto, Brazil

${ }^{\mathrm{b}}$ Department of Neuroscience and Biomedical Engineering, Aalto University School of Science, Espoo, Finland

${ }^{c}$ Graduate Program in Rehabilitation Sciences and Functional Physical Performance, School of Physiotherapy, Federal University of Juiz de Fora, Juiz de Fora, Brazil

${ }^{d}$ Neuro Biomechanics Group, School of Physiotherapy, Federal University of Juiz de Fora, Juiz de Fora, Brazil

e Centre for Cognition and Decision Making, Institute for Cognitive Neuroscience, National Research University Higher School of Economics, Moscow, Russia

${ }^{f}$ Federal State Budgetary Institution «Federal center of brain research and neurotechnologies» of the Federal Medical Biological Agency, Moscow, Russian Federation

*Corresponding author:

Gabriela Pazin Tardelli, Department of Physics, School of Philosophy Science and Literature of Ribeirão Preto, University of São Paulo, Ribeirão Preto, Brazil, email: gabriela.tardelli@usp.br

$\dagger$ These authors have contributed equally to this work and share the first authorship.

\section{Keywords}

Motor mapping, Transcranial magnetic stimulation, TMS, Motor evoked potential, Neuronavigation, Synergy 


\section{Abstract}

Most of the motor mapping procedures using navigated transcranial magnetic stimulation (nTMS) follow the conventional somatotopic organization of the primary motor cortex (M1) by assessing the representation of a particular target muscle, disregarding the possible coactivation of synergistic muscles. In turn, multiple reports describe a functional organization of the M1 with an overlapping among motor representations acting together to execute movements. In this context, the overlap degree among cortical representations of synergistic hand and forearm muscles remains an open question. This study aimed to evaluate the muscle coactivation and representation overlapping common to the grasping movement and its dependence on the stimulation parameters. The nTMS motor maps were obtained from one carpal muscle and two intrinsic hand muscles during rest. We quantified the overlapping motor maps in size (area and volume overlap degree) and topography (similarity and centroid's Euclidean distance) parameters. We demonstrated that these muscle representations are highly overlapped and similar in shape. The overlap degrees involving the forearm muscle were significantly higher than only among the intrinsic hand muscles. Moreover, the stimulation intensity had a stronger effect on the size compared to the topography parameters. Our study contributes to a more detailed cortical motor representation towards a synergistic, functional arrangement of M1. Understanding the muscle group coactivation may provide more accurate motor maps when delineating the eloquent brain tissue during pre-surgical planning.

\section{Introduction}

A fundamental debate on primary motor cortex (M1) organization is whether different body parts rely on a discrete somatotopic or functionally-specific representation on the cortical surface (Schieber 2001). In the M1, the somatotopic organization associates a cortical site to the control of a specific muscle (Penfield and Rasmussen 1950), whereas the functional organization suggests the cortical representation of limb movements (Gentner et al. 2010; Strother et al. 2012). Several studies have demonstrated that the high complexity of central movement generation can be derived from an extensive overlap and redundancy between adjacent cortical area representations (Schieber 2001; Devanne et al. 2006; Gentner and Classen 2006; Melgari et al. 2008). The overlapping areas can be related to specific movements involving more than one adjacent single joint and, therefore, a complex synergy among different muscles (Strother et al. 2012; Leo et al. 2016), which corroborates a hypothesis of the functional organization of $\mathrm{M} 1$. In this context, the overlap degree (OD) in the cortical representation of synergistic hand and forearm muscles remains an open question.

In navigated transcranial magnetic stimulation (nTMS), a coil placed on the scalp over M1 produces magnetic pulses that induce electric fields in the cortical tissue. The neuronal excitation results in action potentials that propagate through the corticospinal tract generating motor evoked potentials (MEP). The MEP amplitude combined with the TMS coil coordinates of the individual's brain enables one to delimit the extension and location of the motor cortical representations of the body parts (Romero et al. 2011). The nTMS mapping is widely used for delineating eloquent motor function in a preoperative setting (Lefaucheur and Picht 2016; Krieg et al. 2017). An approach that accounts for the functional overlap in cortical motor representations can lead to more selective cortical maps with the potential to improve patient prognostics (Frey et al. 2014; Picht et al. 2016).

A few studies claim that the overlap in cortical representation may partially represent a cortical manifestation for synergies (Pearce et al. 2000; Tyc and Boyadjian 2005; Latash et al. 
2007; Cheung et al. 2012; Overduin et al. 2012; Leo et al. 2016; Huffmaster et al. 2018; Raffin and Siebner 2019). In this model, multiple muscle groups form different synergy patterns producing complex movements (Fricke et al. 2020). To the best of our knowledge, most conventional motor mapping procedures assess the cortical representation of a particular target muscle (Krieg et al. 2017), disregarding the possible synergistic activation of the adjacent muscles. This coactivation of muscle groups may provide further information about how and to what degree synergistic muscles are represented at the cortical level (Leo et al. 2016).

The aim of our study was to quantify the OD between the cortical motor representation of two intrinsic hand muscles and one carpal forearm muscle in rest conditions. Using nTMS mapping, we delineated the motor representation of the selected muscles considering their synergistic activation. We hypothesized that these representations would be highly overlapped due to the muscles' extensive coactivation in several hand movements, e.g., grasping. Also, the OD would differ between adjacent and target muscles from different body parts and would increase with the TMS intensity. Our results provide novel evidence on the functional cortical motor organization of the human brain.

\section{Material and Methods}

Participants

The experiment was performed with 12 right-handed (Edinburgh handedness inventory (Oldfield 1971), mean score: +75 ; range: +55 to +95 ) young male volunteers (mean age: $31.3 \pm 2.5$ years; range: $27-35$ years). Participants were asymptomatic to neurological and psychiatric disorders, without recurrent headaches, and free of medication during the data collection phase. The experimental procedure followed the Declaration of Helsinki and was approved by the local ethical committee (CAAE: 54674416.9.0000.5407). Before the testing procedures, all participants signed a consent form.

\section{Experimental procedure}

Subjects underwent a magnetic resonance imaging (MRI) scan (Achieva 3T; Philips Healthcare,

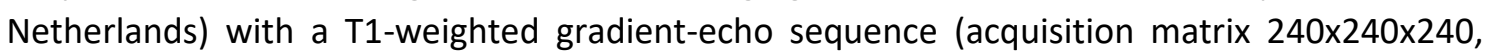
voxel size $1 \times 1 \times 1 \mathrm{~mm}^{3}, 6.7 \mathrm{~ms}$ repetition time, and $3.1 \mathrm{~ms}$ echo time). The gray matter surface of the brain was segmented using SPM 12 software (Friston et al. 2006) for guiding the nTMS coil placement. Surface EMG electrodes (circular 10-mm diameter; model 2223 BRQ, 3M Brazil Ltd., Sumaré, Brazil) were placed in a pseudo-monopolar montage, with one electrode over the innervation zone and the other over the closest bone eminence (Garcia et al. 2017, 2020). The selected muscles were one right carpal forearm muscle (flexor carpi radialis; FCR), and two right intrinsic hand muscles, a thenar (flexor pollicis brevis; FPB) and a hypothenar muscle (abductor digiti minimi; ADM). EMG data were continuously recorded from the three muscles and digitized with the EMG $410 \mathrm{C}$ amplifier (gain: $2000 \mathrm{x}$, sampling frequency: $3.5 \mathrm{kHz}$ per channel, band-pass $4^{\text {th }}$-order Butterworth filter: $20-500 \mathrm{~Hz}, \mathrm{~A} / \mathrm{D}$ converter: 12 bits; EMG System do Brasil, São José dos Campos, Brazil).

The participants sat in a reclining chair and were instructed to stay fully relaxed with their right hand in a neutral posture during the nTMS session. TMS biphasic pulses were delivered with a figure-of-eight coil (10 cm diameter windings) connected to a Neuro-MS stimulator (Neurosoft, Ivanovo, Russia). The coil placement was guided by the neuronavigation software InVesalius Navigator (Souza et al. 2018) connected to the MicronTracker Sx60 (ClaroNav, Toronto, Canada) spatial tracker. Figure $1 \mathrm{~A}$ depicts the experimental setup. The following procedure 
was applied separately for each muscle (FCR, FPB, and ADM). First, the hotspot was defined as the coil location showing the highest MEP amplitudes with the coil tangential to the scalp and approximately perpendicular to the central sulcus (Bashir et al. 2013; Souza et al. 2017). Second, the resting motor threshold (rMT) was defined as the minimum stimulator intensity at which the MEP amplitudes were greater than $100 \mu \mathrm{V}$ in 5 out of 10 pulses (Nielsen 1996; Nogueira-Campos et al. 2014). A higher threshold amplitude than the usual $50 \mu \mathrm{V}$ (Conforto et al. 2004) was selected to provide stable MEP measurements desired when using a relatively small number of trials per stimulation site during motor mapping (Pellegrini et al. 2018).

A

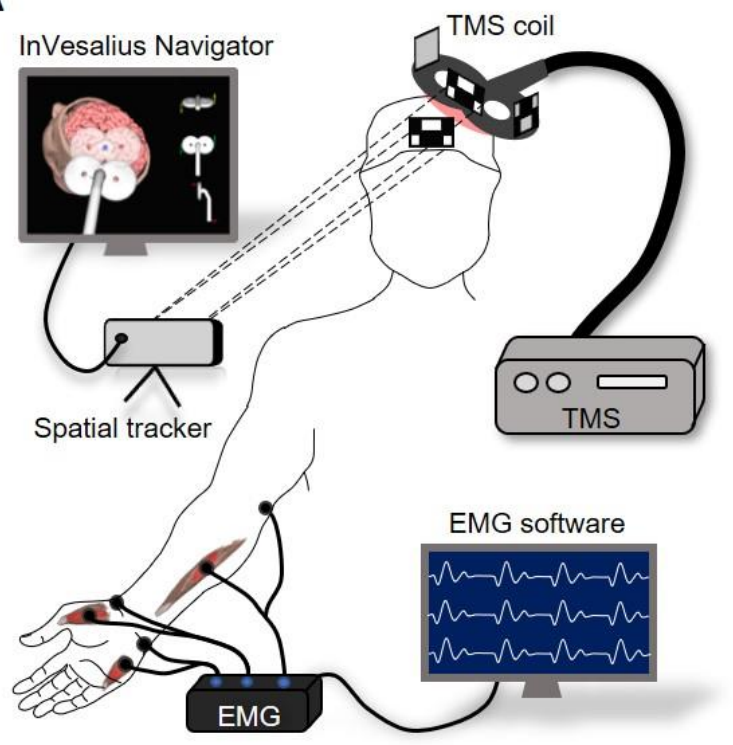

B

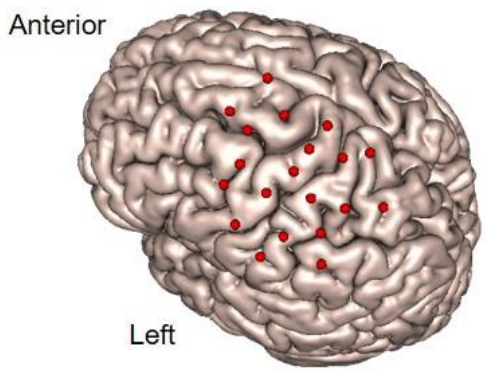

Fig. 1 A) Schematic representation of the experimental setup. The MEPs were recorded simultaneously from the hand and forearm muscles while TMS was applied over the left hemisphere guided by the InVesalius Navigator software. B) Grid of coil center locations relative to the cortical surface. This motor map was recorded at a stimulation intensity of $120 \%$ of rMT of the FCR muscle on a representative subject. The grid was centered on the muscle's hotspot, and each coil location is the average across three trials.

Motor mapping was performed following the pseudo-random walk method with three consecutive TMS pulses in each of the 20 sites around the target muscle's hotspot (Van De Ruit et al. 2015; Cavaleri et al. 2018; Jonker et al. 2018). Each motor map composed an unevenly spaced grid centered on the hotspot for each individual, as illustrated for a representative subject in Fig 1B. The distance between adjacent stimulation sites was approximately $14.5 \pm$ $3.6 \mathrm{~mm}$, and interpulse intervals were pseudo-randomized between 5 and $10 \mathrm{~s}$. EMG signal was recorded from the three muscles simultaneously, and the coordinates of the coil center were recorded with the neuronavigation software. A trigger signal synchronized the EMG and the neuronavigation software. The target muscle was defined as the one whose stimulation intensity was set relative to its $\mathrm{rMT}$, and the stimuli were delivered over a region centered at the hotspot. The remaining muscles were defined as adjacent. The experiment was repeated for each target muscle (ADM, FCR, and FPB) and with stimulation intensities of $110 \%$ and $120 \%$ of the $\mathrm{rMT}$, scaled according to the maximum stimulator output (MSO).

\section{Motor map processing}

The EMG signals were processed using the Signal Hunter software (https://doi.org/10.5281/zenodo.1326308) written in Matlab R2017a (MathWorks Inc., Natick, 
USA). The peak-to-peak amplitude was computed for MEPs extracted from the EMG signal in a time window 10-60 ms after the TMS pulse. The EMG signal was visually inspected, and trials with muscle pre-activation, artifacts, or noise over $\pm 20 \mu \mathrm{V}$ up to $300 \mathrm{~ms}$ before the TMS pulse in amplitude were rejected. After the preprocessing, all subjects had three trials in each of the 20 cortical targets per motor map, except for one subject in which three out of the 60 stimulations were rejected due to muscle pre-activation and noisy EMG signals. The coil center coordinates obtained from InVesalius Navigator were imported into Signal Hunter and aligned to the corresponding MEP amplitude and the latency of the three muscles. One participant was removed from the data analysis due to technical problems in the TMS-EMG synchronization.

The cortical motor maps were created in the TMSmap software (Novikov et al. 2018) with the individuals' MRI, stimulation coordinates, and the correspondent MEP amplitudes. The software creates the maps with the mean coordinates and the median peak-to-peak MEP amplitudes of the merged closely spaced coordinates, resulting in a cortical motor map with 20 MEP amplitudes and coil locations per tested condition for each subject. The technical details of the map processing steps performed in the TMSmap software are described in Appendix 1. For each target muscle, we constructed two overlaps: the target with each of the adjacent muscles (two maps) and the target with both adjacent muscles together (three maps). The area, volume, and centroid were computed for all maps from each subject and stimulation intensity. The area represents the extent of the cortical motor representation, and the volume represents the area weighted by the motor response amplitude. To quantify the topographic similarity between two (or three) maps, we computed the Earth's movers distance (EMD), which estimates the work required to move one spatial distribution to another (Rubner et al. 1998). The Euclidean distance between the target muscle and the overlap map centroids of the corresponding target and its adjacent muscles was computed to evaluate differences in the spatial distribution of the muscle of interest when overlapped with another map. We defined the EMD and the Euclidean distance as topography parameters.

To evaluate the coactivation between the muscle representations, we defined the size parameters as area and volume OD. The OD was computed as the percentage of the area (or volume) that evoked two or three muscles relative to the total area (or volume) that evoked at least one of the assessed muscles (Melgari et al. 2008; Nazarova et al. 2021):

$$
\mathrm{X}_{\mathrm{OD}-2 \text { muscles }}=\frac{\mathrm{X}_{12} \cdot 100 \%}{\mathrm{X}_{1}+\mathrm{X}_{2}-\mathrm{X}_{12}}
$$

Where $\mathrm{X}$ can be area or volume, and the indices 1, 2, and 3 (equation below) refer to each of the muscle maps (target and adjacent) and their corresponding overlaps (pairs of indices). The OD was categorized as: 0-20\% (negligible); $21-40 \%$ (low); $41-60 \%$ (medium); $61-80 \%$ (high); $81-$ $100 \%$ (very high). The relative number of subjects with OD in each of these categories was calculated for all overlap maps. Similarly, the OD of three muscle motor maps were computed as:

$$
\mathrm{X}_{\mathrm{OD}-3 \text { muscles }}=\frac{\mathrm{X}_{123} \cdot 100 \%}{\mathrm{X}_{1}+\mathrm{X}_{2}+\mathrm{X}_{3}-\left(\mathrm{X}_{12}+\mathrm{X}_{13}+\mathrm{X}_{23}\right)+\mathrm{X}_{123}}
$$

\section{Statistical analysis}

All parameters were normalized relative to their maximum values within each individual to enable a direct comparison between conditions and subjects. The stimulation intensity, target, and adjacent muscle and each map parameter (area and volume OD, EMD, and the centroid 
Euclidean distance) were modeled as fixed effects. In turn, the subjects were modeled as a random effect in a linear mixed-effects model. A random structure of the model was selected based on hierarchical sequential testing with each model fit using likelihood-ratio tests. The chosen model was recomputed using restricted maximum likelihood estimation and the $p$ values for fixed effects derived with Satterthwaite approximations in a Type III Analysis of Variance table. When appropriate, posthoc multiple comparisons were performed with estimated marginal means with $p$-value correction for the false discovery rate. The rMT across subjects (random) and muscles (fixed) were also analyzed using a linear mixed-effects model, and multiple comparisons were performed using the Tukey simultaneous tests for the difference of means. Critical deviations from normality were assessed with the residuals' $Q-Q$ plots, and homoscedasticity was inspected with a standard versus fitted values plot. Statistical analysis was performed in R 3.6 (R Core Team, Vienna, Austria) using the Ime4 1.1, afex 0.25 packages, and emmeans 1.4 packages. The level of statistical significance was set at 0.05 .

\section{Results}

Overlap degree and rMT

The rMT varied across muscles $(p=0.039)$ and within subjects $(p=0.015)$, being higher for the FCR compared to the FPB muscle $(p=0.042)$ and similar when comparing ADM with FCR $(p=$ $0.120)$ or FPB $(p=0.852)$ muscles. We computed nine overlap maps for each subject: six overlap maps of two muscles and three overlaps of three muscles. The subscript $[t g]$ refers to the target and $[a d j]$ to the adjacent muscles. The relative number of subjects for each OD category is illustrated in Figure 2. More than $60 \%$ of the subjects had medium to very high area OD but negligible to low volume OD. Maps of two muscles had more subjects with higher ODs than those of three muscles at both stimulation intensities. The number of subjects with high area OD of all maps was higher at $120 \%$ than at $110 \%$ rMT stimulation intensity. 
Overlap degree: $\square$ Negligible (0 - 20\%) $\square$ Low (21 - 40\%) $\square$ Medium (41 - 60\%)

High (61 - 80\%) Wery high (81-100\%)

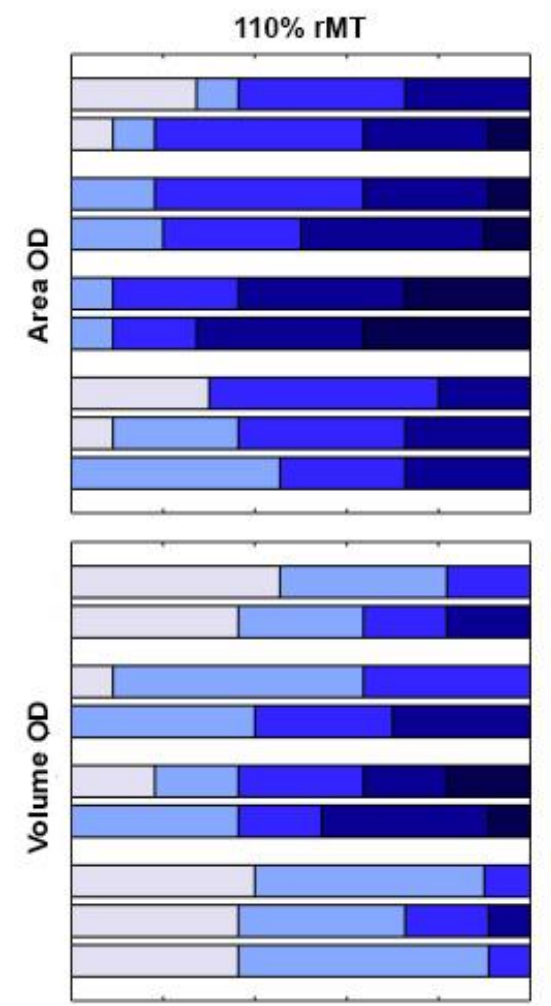

$\begin{array}{lllllll}0 & 20 & 40 & 60 & 80 & 100 & 0\end{array}$
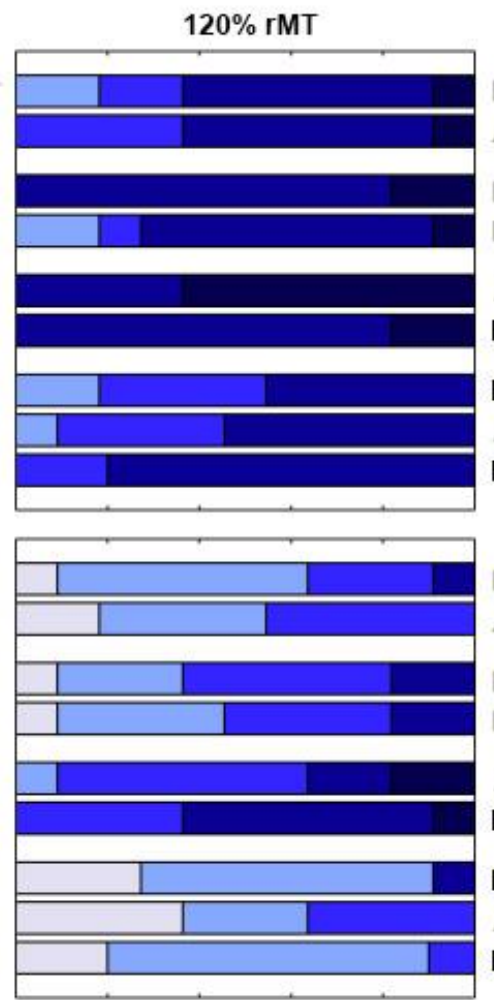

$20 \quad 40$

60

80

$\mathrm{FPB}_{\mathrm{tg}} \cap \mathrm{ADM}_{\mathrm{ad}}$ $\mathrm{ADM}_{\mathrm{tg}} \cap \mathrm{FPB}_{\text {ad] }}$ $\mathrm{FCR}_{\mathrm{tg}} \cap \mathrm{FPB}_{\text {ad] }}$ $\mathrm{FPB}_{\text {tg }} \cap \mathrm{FCR}_{\text {ad] }}$ $\mathrm{ADM}_{\mathrm{tg}} \cap \mathrm{FCR}_{\mathrm{dj}}$ $\mathrm{FCR}_{\mathrm{ig}} \cap \mathrm{ADM}_{\mathrm{ag}}$ $\mathrm{FPB}_{\mathrm{tg}} \cap \mathrm{ADM}_{\mathrm{add}} \cap \mathrm{FCR}_{\mathrm{ad}}$ $\mathrm{ADM}_{\mathrm{tg}} \cap \mathrm{FPB}_{\text {ad] }} \cap \mathrm{FCR}_{\text {ad] }}$ $\mathrm{FCR}_{\mathrm{tg}} \cap \mathrm{FPB}_{\mathrm{add}} \cap \mathrm{ADM}_{\mathrm{ad}}$

$\mathrm{FPB}_{\mathrm{tg}} \cap \mathrm{ADM} \mathrm{M}_{\mathrm{ad}}$ $\mathrm{ADM}_{\mathrm{tg}} \cap \mathrm{FPB}_{\mathrm{ad}]}$ $\mathrm{FCR}_{\mathrm{tg}} \cap \mathrm{FPB}_{\mathrm{ad}]}$ $\mathrm{FPB}_{\mathrm{tg}}^{\mathrm{tg}} \cap \mathrm{FCR}_{\mathrm{ad}]}$ $\mathrm{ADM}_{\mathrm{tg}} \cap \mathrm{FCR}_{\mathrm{ol}}$ $\mathrm{FCR}_{\mathrm{vg}} \cap \mathrm{ADM}_{\mathrm{aq}}$ $\mathrm{FPB}_{\mathrm{tg}} \cap \mathrm{ADM}_{\mathrm{adj}} \cap \mathrm{FCR}_{\text {ad }}$ $\mathrm{ADM}_{\mathrm{tg}} \cap \mathrm{FPB}_{\mathrm{ad} \mid} \cap \mathrm{FCR}_{\mathrm{ad}}$ $\mathrm{FCR}_{\text {tg }} \cap \mathrm{FPB}_{\text {ad] }} \cap \mathrm{ADM}_{\text {adg }}$

Relative number of subjects (\%)

Fig. 2 Relative number of subjects with OD distributed in 5 categories (negligible, low, medium, high and very high) for all overlap maps and at stimulation intensities of $110 \%$ (left) and $120 \%$ (right) of rMT. The length of the horizontal bars indicates the relative number of subjects with each $\mathrm{OD}$, and the $\cap$ (intersection) symbol represents the overlap between muscles.

The effect of stimulation intensity, target and adjacent muscle on map size and topography Table 1 presents the results of the linear mixed-effects model. The size parameters (area and volume OD) were significantly affected by the stimulation intensity, the target and adjacent muscle individually, and their interaction. In turn, the topography parameters (EMD and the centroid Euclidean distance) were significantly affected only by the adjacent muscle but not by the stimulation intensity nor the target muscle. The interaction between stimulation intensity and target muscle affected both topography parameters, while the interaction between target and adjacent muscles affected only the EMD. 
Table 1 The linear mixed-effects model results for size (area and volume OD) and topography (EMD and centroid Euclidean distance) parameters. Abbreviations: degrees of freedom (DoF), stimulation intensity $(\mathrm{SI})$, target $\left(\mathrm{M}_{\mathrm{tg}}\right)$, and adjacent muscle $\left(\mathrm{M}_{\mathrm{adj}}\right)$. The ${ }^{*}$ indicates $p$-value $<$ 0.05

\begin{tabular}{|c|c|c|c|c|c|c|}
\hline Effect & $\begin{array}{l}\text { DoF } \\
\text { (numerator, } \\
\text { denominator) }\end{array}$ & F-value & $p$-value & $\begin{array}{l}\text { DoF } \\
\text { (numerator, } \\
\text { denominator) }\end{array}$ & F-value & $p$-value \\
\hline & \multicolumn{3}{|c|}{ Area OD } & \multicolumn{3}{|c|}{ Volume OD } \\
\hline SI & $(1,10.0)$ & 19.99 & $0.001^{*}$ & $(1,9.96)$ & 5.85 & $0.036^{*}$ \\
\hline$M_{\mathrm{tg}}$ & $(2,10.7)$ & 8.61 & $0.006^{*}$ & $(2,10.9)$ & 11.82 & $0.002^{*}$ \\
\hline$M_{\text {adj }}$ & $(3,137.4)$ & 45.77 & $<0.001^{*}$ & $(3,136.0)$ & 70.35 & $<0.001^{*}$ \\
\hline $\mathrm{SI} \times \mathrm{M}_{\mathrm{tg}}$ & $(2,137.9)$ & 0.82 & 0.444 & $(2,136.6)$ & 0.06 & 0.945 \\
\hline SI x $\mathbf{M}_{\text {adj }}$ & $(3,137.4)$ & 0.09 & 0.964 & $(3,135.9)$ & 1.11 & 0.348 \\
\hline$M_{\mathrm{tg}} \times M_{\mathrm{adj}}$ & $(3,137.4)$ & 7.02 & $<0.001^{*}$ & $(3,136.0)$ & 13.01 & $0.000^{*}$ \\
\hline \multirow[t]{2}{*}{$S I \times M_{t g} \times M_{a d j}$} & $(3,137.5)$ & 1.30 & 0.276 & $(3,136.0)$ & 0.70 & 0.553 \\
\hline & \multicolumn{3}{|c|}{ Centroid Euclidean distance } & \multicolumn{3}{|c|}{ EMD } \\
\hline SI & $(1,11.0)$ & 0.02 & 0.890 & $(1,10.1)$ & 1.65 & 0.228 \\
\hline $\mathrm{M}_{\mathrm{tg}}$ & $(2,11.1)$ & 0.39 & 0.688 & $(2,14.5)$ & 2.76 & 0.096 \\
\hline$M_{\text {adj }}$ & $(3,146.9)$ & 4.49 & $0.005^{*}$ & $(3,147.5)$ & 5.57 & $0.001^{*}$ \\
\hline $\mathrm{SI} \times \mathrm{M}_{\mathrm{tg}}$ & $(2,147.1)$ & 4.06 & $0.019 *$ & $(2,147.9)$ & 3.16 & $0.045^{*}$ \\
\hline SI $x M_{a d j}$ & $(3,146.9)$ & 0.31 & 0.819 & $(3,147,4)$ & 0.28 & 0.836 \\
\hline$M_{\mathrm{tg}} \times M_{\mathrm{adj}}$ & $(3,146.9)$ & 1.32 & 0.270 & $(3,147.5)$ & 4.82 & $0.003 *$ \\
\hline SI $\times M_{t g} \times M_{\text {adj }}$ & $(3,146.9)$ & 1.41 & 0.241 & $(3,147.6)$ & 0.55 & 0.646 \\
\hline
\end{tabular}

The multiple comparisons are presented in Tables 2-4, and the means and standard deviations across subjects for each parameter of all overlap maps area are illustrated in Figure 3. For most overlaps, the area OD was significantly higher at $120 \%$ than at $110 \%$ rMT of stimulation intensity. The intensity effect was only significant at the volume OD factor level, whereas none of the multiple comparisons displayed any significant differences. When comparing different target or adjacent muscles, the highest area and volume ODs were between ADM and FCR. In turn, overlaps involving FPB did not show any significant differences when compared to the overlap between the three muscles together. This result applies to both stimulation intensities in most cases. Lastly, the EMD and centroid Euclidean distance were similar for both tested stimulation intensities and all comparisons across adjacent and target muscles. 
Table 2 Multiple comparisons of area OD, volume OD, centroid's Euclidean distance, and EMD between the stimulation intensities ( $110 \%$ compared with $120 \%$ of $\mathrm{rMT}$ ) for each combination of target and adjacent muscles.

\begin{tabular}{|c|c|c|c|c|c|c|c|c|}
\hline \multirow{3}{*}{$\begin{array}{l}\text { Target } \\
\text { Muscle }\end{array}$} & \multicolumn{8}{|c|}{ Adjacent Muscle } \\
\hline & \multicolumn{4}{|c|}{ Area OD } & \multicolumn{4}{|c|}{ Volume OD } \\
\hline & ADM & FPB & FCR & ALL & ADM & FPB & FCR & ALL \\
\hline ADM & $x$ & 0.937 & $0.016^{*}$ & 0.062 & $x$ & 1.000 & 0.411 & 1.000 \\
\hline FPB & $0.003 *$ & $x$ & 0.073 & $0.006^{*}$ & 0.074 & $x$ & 1.000 & 0.327 \\
\hline \multirow[t]{3}{*}{ FCR } & $0.044^{*}$ & $0.003 *$ & $x$ & $0.004 *$ & 0.090 & 0.573 & $x$ & 1.000 \\
\hline & \multicolumn{4}{|c|}{ Centroid's Euclidean Distance } & \multicolumn{4}{|c|}{ EMD } \\
\hline & ADM & FPB & FCR & $\overline{A L L}$ & ADM & FPB & FCR & $\overline{A L L}$ \\
\hline ADM & $x$ & 0.698 & 1.000 & 0.522 & $x$ & 1.000 & 1.000 & 1.000 \\
\hline FPB & 1.000 & $x$ & 1.000 & 1.000 & 0.587 & $x$ & 1.000 & 0.588 \\
\hline FCR & 1.000 & 1.000 & $x$ & 0.925 & 1.000 & 0.588 & $x$ & 0.588 \\
\hline
\end{tabular}

Table 3 Multiple comparisons of area OD, volume OD, centroid's Euclidean distance, and EMD between different target muscles for each adjacent muscle and stimulation intensity (\% of rMT).

\begin{tabular}{|c|c|c|c|c|c|c|c|c|c|c|}
\hline & \multirow{2}{*}{\multicolumn{2}{|c|}{ Muscles }} & \multicolumn{2}{|c|}{ Area OD } & \multicolumn{2}{|c|}{ Volume OD } & \multicolumn{2}{|c|}{$\begin{array}{c}\text { Centroid's } \\
\text { Euclidean } \\
\text { distance }\end{array}$} & \multicolumn{2}{|c|}{ EMD } \\
\hline \multirow[b]{2}{*}{ Target 1} & & & \multicolumn{8}{|c|}{ Intensity (\% rMT) } \\
\hline & $\begin{array}{c}\text { Target } \\
2\end{array}$ & Adjacent & 110 & 120 & 110 & 120 & 110 & 120 & 110 & 120 \\
\hline FPB & FCR & ADM & $0.001^{*}$ & $0.006^{*}$ & $<0.001^{*}$ & $<0.001^{*}$ & 1.000 & 1.000 & 0.053 & 0.157 \\
\hline ADM & FCR & FPB & 1.000 & 0.102 & 1.000 & 0.662 & 0.878 & 0.826 & 1.000 & 0.253 \\
\hline ADM & FPB & FCR & $0.011^{*}$ & $0.003^{*}$ & 0.444 & 0.115 & 1.000 & 1.000 & 0.697 & 1.000 \\
\hline ADM & FPB & ALL & 0.212 & 0.890 & 0.513 & 1.000 & 1.000 & 0.826 & 1.000 & 1.000 \\
\hline ADM & FCR & ALL & 1.000 & 0.434 & 1.000 & 1.000 & 0.826 & 0.801 & 1.000 & 1.000 \\
\hline FPB & FCR & ALL & 0.307 & 0.230 & 0.513 & 1.000 & 1.000 & 1.000 & 1.000 & 1.000 \\
\hline
\end{tabular}


Table 4 Multiple comparisons of area OD, volume OD, centroid's Euclidean distance, and EMD between different adjacent muscles for each target muscle and stimulation intensity (\% of rMT).

\begin{tabular}{|c|c|c|c|c|c|c|c|c|c|c|}
\hline & \multirow{2}{*}{\multicolumn{2}{|c|}{ Muscles }} & \multicolumn{2}{|c|}{ Area OD } & \multicolumn{2}{|c|}{ Volume OD } & \multicolumn{2}{|c|}{$\begin{array}{l}\text { Centroid's } \\
\text { Euclidean } \\
\text { distance }\end{array}$} & \multicolumn{2}{|c|}{ EMD } \\
\hline \multirow[b]{2}{*}{ Target } & & & \multicolumn{8}{|c|}{ Intensity (\% rMT) } \\
\hline & $\begin{array}{c}\text { Adjacent } \\
1\end{array}$ & $\begin{array}{c}\text { Adjacent } \\
2\end{array}$ & 110 & 120 & 110 & 120 & 110 & 120 & 110 & 120 \\
\hline ADM & FPB & FCR & $0.001^{*}$ & $<0.001^{*}$ & $<0.001 *$ & $<0.001^{*}$ & 1.000 & 0.522 & 1.000 & 0.253 \\
\hline ADM & FPB & ALL & 0.309 & 0.503 & 0.444 & 0.345 & 1.000 & 1.000 & 1.000 & 0.209 \\
\hline ADM & FCR & ALL & $<0.001^{*}$ & $<0.001^{*}$ & $<0.001^{*}$ & $<0.001^{*}$ & 1.000 & 0.449 & 1.000 & 1.000 \\
\hline FPB & ADM & FCR & 0.062 & 1.000 & $<0.001^{*}$ & 0.066 & 0.826 & 1.000 & 0.209 & 0.588 \\
\hline FPB & ADM & ALL & 0.503 & 0.212 & 0.899 & 0.327 & 1.000 & 1.000 & 0.109 & 0.086 \\
\hline FPB & FCR & ALL & $0.004^{*}$ & 0.083 & $<0.001^{*}$ & $<0.001^{*}$ & 0.826 & 1.000 & 1.000 & 0.733 \\
\hline FCR & ADM & FPB & $0.001 *$ & $0.044^{*}$ & $0.001 *$ & $<0.001^{*}$ & 0.522 & 0.925 & 0.253 & 1.000 \\
\hline FCR & ADM & ALL & $<0.001^{*}$ & $<0.001^{*}$ & $<0.001^{*}$ & $<0.001^{*}$ & 0.463 & 0.878 & 0.697 & 1.000 \\
\hline FCR & FPB & ALL & 0.096 & 0.086 & $0.042 *$ & $0.003^{*}$ & 1.000 & 1.000 & 1.000 & 1.000 \\
\hline
\end{tabular}


Target muscle
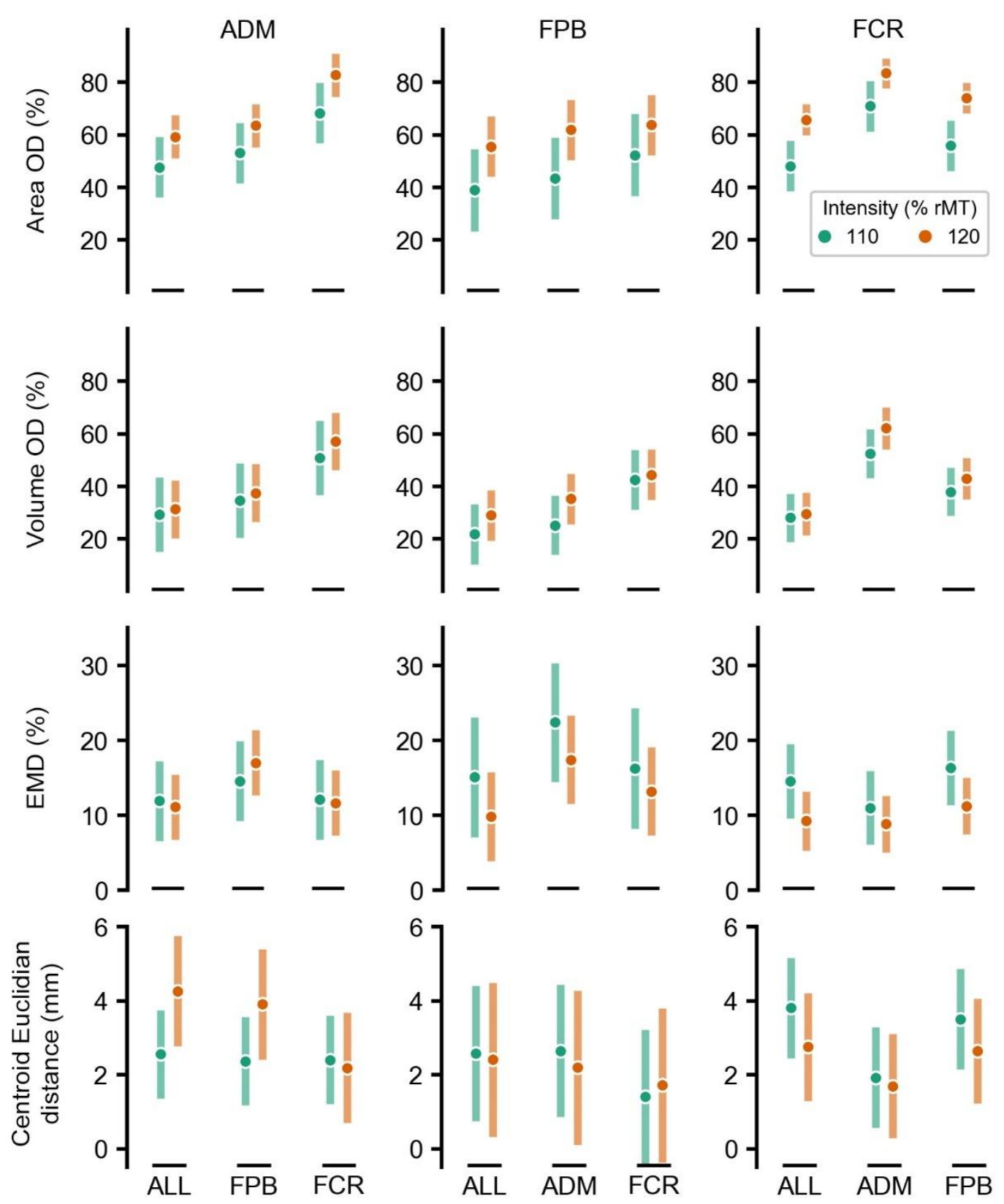

Adjacent muscle

Fig. 3 Mean and standard deviation of the motor maps' size and topography parameters across the 11 subjects. The rows and columns of the chart grid contain the map parameters and the target muscle, respectively. The green and orange colors represent $110 \%$ and $120 \%$ of the rMT stimulation intensity. The term ALL refers to the overlap of the target with both adjacent muscles simultaneously

\section{Discussion}

In this study, we demonstrate that the motor map parameters vary significantly between the pairs of muscles in size (area and volume) but not in topography (EMD and centroid Euclidean distance). While area OD of the muscle's pairs was mainly medium to very high, volume OD was negligible to low. Our results also show that increasing the stimulation intensity from 
bioRxiv preprint doi: https://doi.org/10.1101/2021.07.20.453121; this version posted November 30, 2021. The copyright holder for this preprint (which was not certified by peer review) is the author/funder, who has granted bioRxiv a license to display the preprint in perpetuity. It is made available under aCC-BY-NC-ND 4.0 International license.

$110 \%$ to $120 \%$ of the rMT causes a significant increase in the area OD among different muscles. In turn, changing the stimulation intensity does not affect the map topographies. The volume $O D$ does not seem to increase with the stimulus intensity, possibly due to OD variations between subjects and muscles.

The effect of TMS intensity on the map parameters

Lower stimulation intensities resulted in a motor representation with more restricted sites of muscle activation than higher intensities, as depicted in Figure 4. One likely interpretation is that the weaker intensity might not recruit the less excitable neuronal populations (Kallioniemi and Julkunen 2016). When studying the functional overlapping of muscles with different motor thresholds, one might observe that M1 is organized by discrete or slightly overlapping representations. In each M1 representation site, the target muscle can be overlapped with different adjacent muscles that may be related to distinct synergies and, therefore, contribute to various movements. This association might point towards the functional organization of M1 (Massé-Alarie et al. 2017).

In turn, higher stimulation intensities are associated with stronger magnetic fields spanning a larger cortical region (van de Ruit and Grey 2016) and reflect in smoother motor maps that may lead to two outcomes. First, the higher intensity may excite neuronal populations located further from the region of interest, recruited indirectly from the excitation of intracortical neurons, thus producing progressively larger motor map areas (Schieber 2001; Nieminen et al. 2019). This stimulation leakage may overestimate the muscle representations and the regionof-interest muscle representation overlap, losing the specificity of the studied movement. Secondly, the higher intensity may delineate the full extent of the muscles' motor representation, providing a complete picture of the muscle group coactivation. In summary, coactivation maps obtained from lower and higher stimulation intensities might provide complementary perspectives on the M1's functional organization. The stimulation intensity needs to be carefully chosen to account for the synergy when mapping the representation of different muscles, especially in pre-surgical applications where the mapping methods must be the most accurate possible (Krieg et al. 2017). 


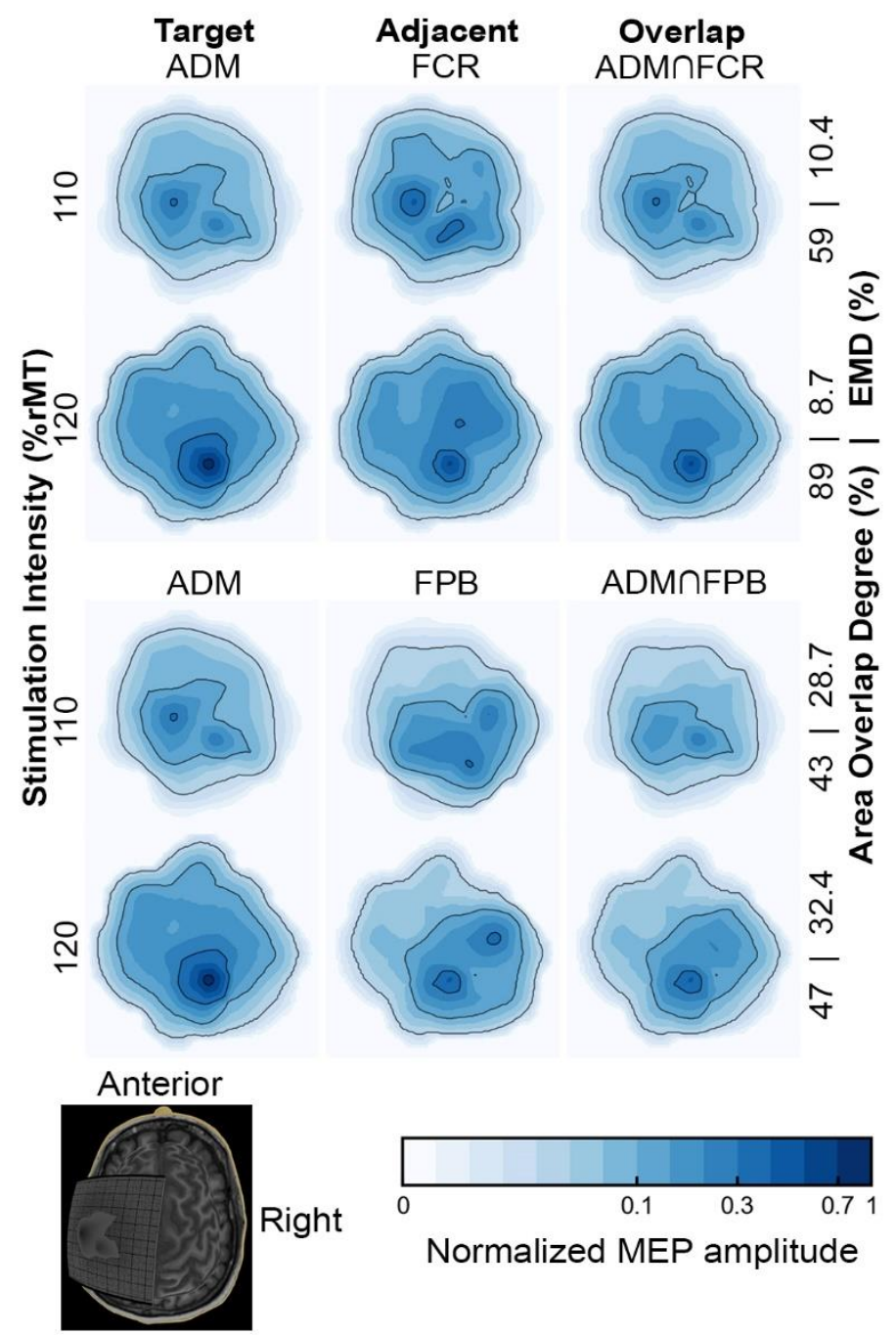

Fig. 4 Normalized motor maps of a representative subject with low and high area and topographic similarity, and MEP amplitude affected by a higher TMS intensity. The contour line indicates the 5,30 , and $70 \%$ of the maximal MEP amplitude. Note that the map size and the area OD, but not the EMD, is significantly higher at $120 \%$ than in $110 \%$ of $\mathrm{rMT}$. In addition, the increase in the area OD is greater when the EMD is lower, i.e., when the maps of the target and adjacent muscles have a more remarkable similarity. The $\cap$ (intersection) symbol represents the overlap between muscles.

A stimulation intensity of $120 \%$ of $\mathrm{rMT}$ resulted in larger representation areas of the target and adjacent muscles, corroborating previous findings (Thordstein et al. 2013; Julkunen 2014; Kallioniemi and Julkunen 2016; van de Ruit and Grey 2016). The increase in the OD is due to a higher spatial overlap between the two cortical maps than in the total area encompassed by two (or three) maps individually, as illustrated in Figure 5. Regardless of the higher overlap, the spatial distributions remain similar, and the most excitable regions of the cortex for a particular muscle seem to stay the same. Our results align with a previous study showing that an increase in stimulation intensity changes the extension of the motor representation but keeps similar topographies and centroids (van de Ruit and Grey 2016). 


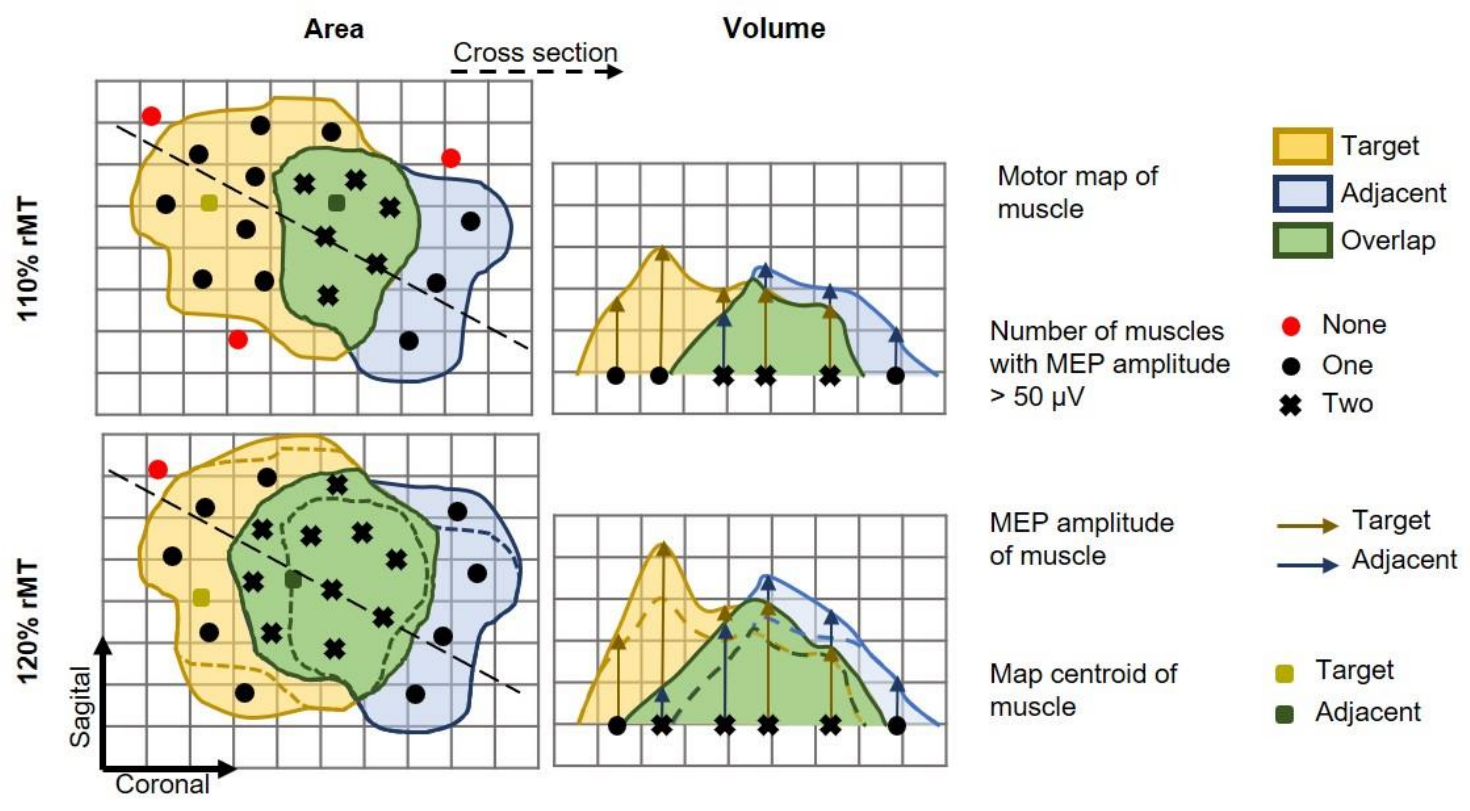

Fig. 5 Schematic illustration of the effect of the stimulation intensity on the individual and overlap maps. In the left panel, the motor map area, and on the right, a cross-section represents the map's height profile (MEP amplitude). Note that, at a higher stimulation intensity $(120 \% \mathrm{rMT})$, the increase in the number of stimulation sites resulting in MEP amplitudes greater than $50 \mu \mathrm{V}$ (black markers) from one (circle) to two (cross) muscles was more pronounced than the increase from none to one muscle. Thus, even though all maps (target and adjacent muscle and overlap) showed an increase in area, the overlap map increased more than the total map

The cortical representation overlapping of a carpal and intrinsic hand muscles

We observed a higher overlapping between the forearm (FCR) and both intrinsic hand muscles ( $A D M$ or $\mathrm{FPB}$ ) than between the intrinsic themselves. In addition, the centroids of the hand muscle representations are further away than those between the forearm and hand muscles. In contrast, a previous study observed higher levels of overlapping among the representations of intrinsic hand muscles when compared to those obtained between them and the carpal forearm muscles (Melgari et al. 2008; Nazarova et al. 2021). Possibly, this difference is because the subjects in our study kept their hands in a neutral posture, offering distinct proprioceptive feedback from that generated in maintaining the pronated posture (Graziano 2006), as adopted in the study by (Melgari et al. 2008). The hand posture is implicitly related to grasping and the corresponding body movements (Perez and Rothwell 2015). The influence of the hand posture on overlapping was previously associated with a dynamic modification in the neuronal network structure related to motor control (Melgari et al. 2008; Perez and Rothwell 2015; Raffin and Siebner 2019). Furthermore, the higher rMT of the forearm compared to the hand muscles may contribute to the observed higher overlap, while the low rMT of FPB may only partially stimulate adjacent muscles with higher rMT. In this sense, our results suggest that different muscles have cortical areas preferentially shared with specific muscles, as depicted in Figure 6. 


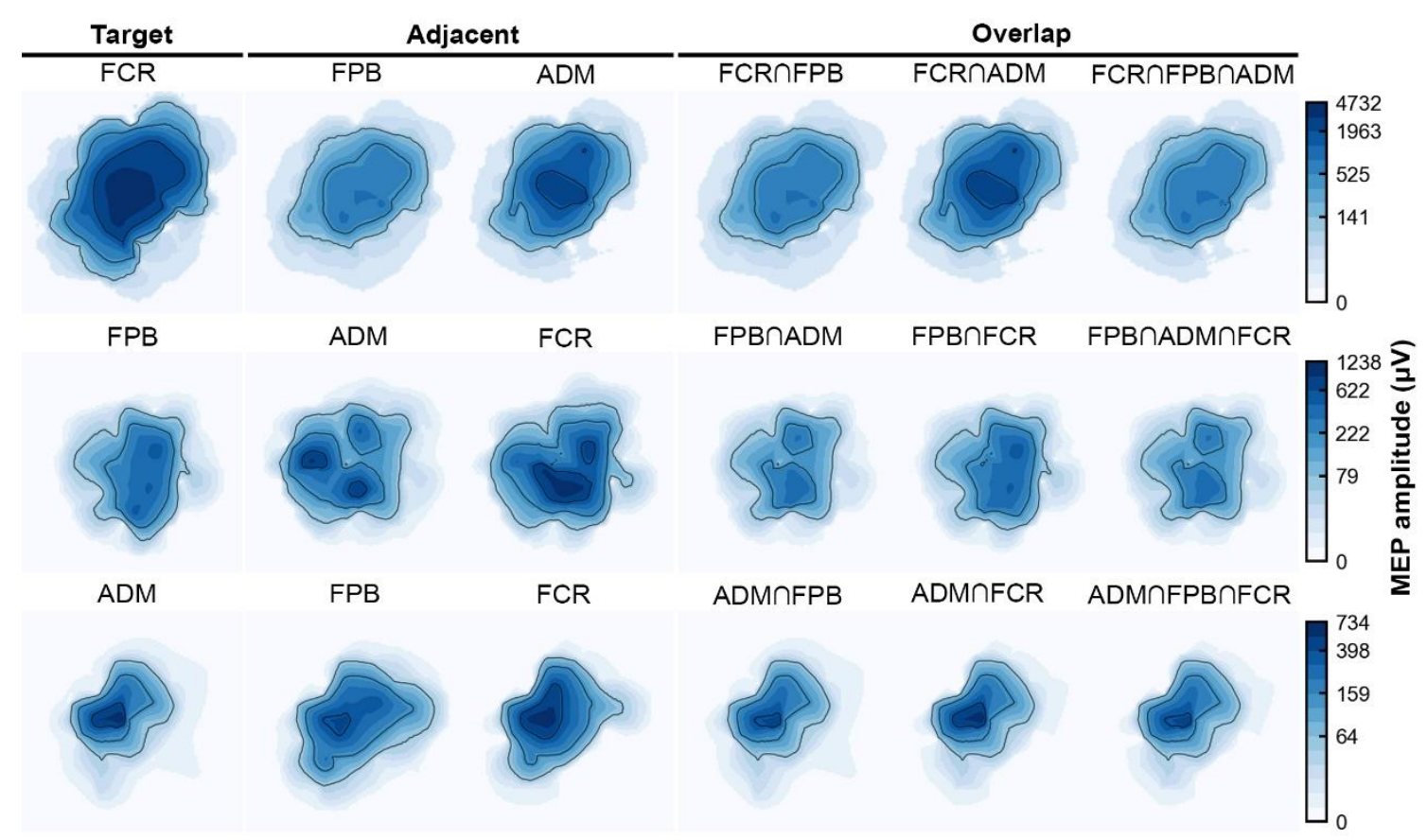

Fig. 6 Motor maps of a representative subject at a stimulation intensity of $120 \%$ rMT, comparing topography, size, and MEP amplitudes of all possible combinations of ADM, FPB, and FCR muscle maps. The map's anatomical references are the same as in Figure 4 . The $\cap$ (intersection) symbol represents the overlap between muscles.

We associate the high overlap in motor representations of upper limb muscles to a functionally organized M1 hypothesis. Our view is in agreement with previous studies that investigated the organization pattern of M1 through the nTMS motor overlapping (Wassermann et al. 1992; Wilson et al. 1993; Marconi et al. 2007; Melgari et al. 2008; Nazarova et al. 2021), and functional MRI in humans (Indovina and Sanes 2001; Leo et al. 2016). As we expected, our results revealed that the OD was smaller but still significant when both hand and forearm muscles were overlapped. This may indicate that increasing the number of overlapped motor representations reduces the overlapping degrees, resulting in greater specificity of the evoked synergies. Despite the highly overlapped representations, we have not focused on whether it reflects the ability to perform fine movements, as previous studies interpreted as indications of functional reorganization of M1 (Pearce et al. 2000; Tyc and Boyadjian 2005). However, this factor could be associated with different OD among subjects and may be tested in the future through parallel behavioral approaches.

The substantial overlapping is possibly explained by how the M1 seems to encode movements and muscle recruitment. Individual muscles appear to be recruited by a complex neuronal network instead of an individualized set of neurons, connected by a set of synergies responsible for a wide range of movements and tasks (Gentner and Classen 2006; Leo et al. 2016). Such motor representation is given by groups of functionally related neurons (Klochkov et al. 2018) following two possible mechanisms: convergence and divergence. In the convergence mechanism, a muscle has its motor representation on separate sites over M1, i.e., each site is probably overlapped with the motor representation of different groups of muscles and, thus, associated with various movements (Schieber 2001; Massé-Alarie et al. 2017). In the divergence mechanism, one site can elicit several muscles simultaneously with different intensities according to their performed movement (Schieber 2001; Melgari et al. 2008). 
bioRxiv preprint doi: https://doi.org/10.1101/2021.07.20.453121; this version posted November 30, 2021. The copyright holder for this preprint (which was not certified by peer review) is the author/funder, who has granted bioRxiv a license to display the preprint in perpetuity. It is made available under aCC-BY-NC-ND 4.0 International license.

The divergence mechanism is observed through connections between specific pyramidal neurons and motoneurons associated with different muscles (Schieber 2001). Such a phenomenon could be related to the shared innervation between FPB with both ADM and FCR. The FPB is a hypothenar muscle composed of a superficial and a deep head that have different innervations. The deep head of the FPB is innervated by the ulnar nerve, the same innervation of the ADM. The superficial head of the FPB has the same innervation as the FCR, the median nerve (Vishram 2014). It possibly relates to the OD in the cortical representation. The action potential generated in the cortical site associated with the FPB propagates through similar pathways, resulting in a simultaneous contraction of the muscles with shared innervations. Therefore, the significant area OD probably resulted from the divergence of the overlapping motor representation indicating the synergism between the studied muscles. Animal studies further support such a view. For instance, cortical motor neurons in cats are connected to neurons of multiple muscles, not as the expected point-to-point connectivity. The synergistic interactions between neuronal populations in different cortical sites might generate descending volleys influencing various movements through the recruitment of multiple muscles (Capaday et al. 2009).

\section{Methodological considerations}

Despite the evident extension of the overlapping representation of muscles on M1, the focality of the TMS is challenging to estimate, and stimulation can propagate over regions responsible for adjacent muscles that can contribute to a high OD (Schieber 2001; Fricke et al. 2017). However, simple models based on the coil's center projection, like the one used in this experiment, have more than $85 \%$ accuracy and can delineate the cortical motor representation, but the stimulus propagation in the realistic cortical geometry is still disregarded (Seynaeve et al. 2019).

We should note that the EMG crosstalk in the pseudo-monopolar montage may contaminate the cortical motor representations. However, the electrode over the innervation zone might detect the direct neural drive to the muscle alleviating the crosstalk for the intrinsic hand muscles (superficial fibers running parallel to the skin) and the FCR muscle we studied (Garcia et al. 2017, 2020). Also, the cortical overlaps between the forearm and intrinsic hand muscle are more prominent than the overlap within intrinsic muscles. Thus, considering that the hand muscles are closely located and have bigger crosstalk between each other (Selvanayagam et al. 2012), our findings are significant despite this limitation and would only be further supported by reducing the potential crosstalk.

We used $100 \mu \mathrm{V}$ MEP amplitude as a reference to estimate the rMT. The $110 \%$ and $120 \%$ of rMT stimulation intensities may correspond to slightly higher stimulator outputs when compared to protocols using $50 \mu \mathrm{V}$ MEP amplitude. Nonetheless, our key results are the changes in motor map parameters and overlap relative to the increase in the stimulation, which are likely to occur regardless of the small deviation from the stimulator output. Moreover, the adopted protocol ensured consistent MEPs for a relatively small number of trials in each location coil location during the motor mapping procedure. We should note that only one set of muscles linked to the manual grasp movement was assessed. Our results may not generalize to muscle groups with different movement refinement, such as the lower limbs. Even so, we provide an important systemic perspective on how to evaluate the cortical motor representation. 


\section{Conclusion}

We aimed at understanding the cortical motor organization of three muscles linked to the grasping movement. Our results showed a higher cortical representation overlapping between the carpal forearm and both intrinsic hand muscles than between the intrinsic themselves. Stronger stimulation intensities led to higher overlap in the map areas but did not affect the volume and the map topographies. Our study contributes to a more detailed representation of the motor cortex associated with the functional arrangement among muscles, implying a synergistic spatial organization. Understanding the coactivation of muscle groups may provide accurate functional maps over M1. Finally, spatially accurate cortical motor mapping with nTMS can have an immediate clinical impact, for instance, when defining the eloquent brain regions during pre-surgical planning. Avoiding highly overlapped areas associated with muscular synergy would minimize deficits in the patients' motor function (Tharin and Golby 2007; Lefaucheur and Picht 2016).

\section{References}

Bashir S, Perez JM, Horvath JC, Pascual-Leone A (2013) Differentiation of Motor Cortical Representation of Hand Muscles by Navigated Mapping of Optimal TMS Current Directions in Healthy Subjects. Journal of Clinical Neurophysiology 30:390-395. https://doi.org/10.1097/WNP.0b013e31829dda6b

Capaday C, Ethier C, Brizzi L, et al (2009) On the nature of the intrinsic connectivity of the cat motor cortex: Evidence for a recurrent neural network topology. Journal of Neurophysiology 102:2131-2141. https://doi.org/10.1152/jn.91319.2008

Cavaleri R, Schabrun SM, Chipchase LS (2018) The reliability and validity of rapid transcranial magnetic stimulation mapping. Brain Stimulation 11:1291-1295. https://doi.org/10.1016/j.brs.2018.07.043

Cheung VCK, Turolla A, Agostini M, et al (2012) Muscle synergy patterns as physiological markers of motor cortical damage. Proceedings of the National Academy of Sciences of the United States of America 109:14652-14656. https://doi.org/10.1073/pnas.1212056109

Conforto AB, Z'Graggen WJ, Kohl AS, et al (2004) Impact of coil position and electrophysiological monitoring on determination of motor thresholds to transcranial magnetic stimulation. Clinical Neurophysiology 115:812-819. https://doi.org/10.1016/j.clinph.2003.11.010

Devanne H, Cassim F, Ethier C, et al (2006) The comparable size and overlapping nature of upper limb distal and proximal muscle representations in the human motor cortex. European Journal of Neuroscience 23:2467-2476. https://doi.org/10.1111/j.14609568.2006.04760.x

Frey D, Schilt S, Strack V, et al (2014) Navigated transcranial magnetic stimulation improves the treatment outcome in patients with brain tumors in motor eloquent locations. NeuroOncology 16:1365-1372. https://doi.org/10.1093/neuonc/nou110

Fricke C, Gentner R, Alizadeh J, Classen J (2020) Linking Individual Movements to a Skilled Repertoire: Fast Modulation of Motor Synergies by Repetition of Stereotyped Movements. Cerebral Cortex 30:1185-1198. https://doi.org/10.1093/cercor/bhz159 
Fricke C, Gentner R, Rumpf JJ, et al (2017) Differential spatial representation of precision and power grasps in the human motor system. Neurolmage 158:58-69. https://doi.org/10.1016/j.neuroimage.2017.06.080

Friston K, Ashburner J, Kiebel S, et al (2006) Statistical Parametric Mapping: The Analysis of Functional Brain Images, 1st Editio. Academic Press

Garcia MAC, Souza VH, Lindolfo-Almas J, et al (2020) Motor potential evoked by transcranial magnetic stimulation depends on the placement protocol of recording electrodes: A pilot study. Biomedical Physics and Engineering Express 6:047003. https://doi.org/10.1088/2057-1976/ab950a

Garcia MAC, Souza VH, Vargas CD (2017) Can the Recording of Motor Potentials Evoked by Transcranial Magnetic Stimulation Be Optimized? Frontiers in Human Neuroscience 11:14. https://doi.org/10.3389/fnhum.2017.00413

Gentner R, Classen J (2006) Modular Organization of Finger Movements by the Human Central Nervous System. Neuron 52:731-742. https://doi.org/10.1016/j.neuron.2006.09.038

Gentner R, Gorges S, Weise D, et al (2010) Encoding of motor skill in the corticomuscular system of musicians. Current Biology 20:1869-1874. https://doi.org/10.1016/j.cub.2010.09.045

Graziano M (2006) The Organization of Behavioral Repertoire in Motor Cortex. The Annual Review of Neuroscience 29:105-134. https://doi.org/10.1146/annurev.neuro.29.051605.112924

Groppa S, Oliviero A, Eisen A, et al (2012) A practical guide to diagnostic transcranial magnetic stimulation: Report of an IFCN committee. Clinical Neurophysiology 123:858-882. https://doi.org/10.1016/j.clinph.2012.01.010

Huffmaster XSLA, lii GMVA, Luchies CW, Cheney XD (2018) Muscle Synergies Obtained from Comprehensive Mapping of the Cortical Forelimb Representation Using Stimulus Triggered Averaging of EMG Activity. The Journal of Neuroscience 38:8759-8771. https://doi.org/10.1523/JNEUROSCI.2519-17.2018

Indovina I, Sanes JN (2001) On somatotopic representation centers for finger movements in human primary motor cortex and supplementary motor area. Neuroimage 13:10271034. https://doi.org/10.1006/nimg.2001.0776

Jonker ZD, Vliet R van der, Hauwert CM, et al (2018) TMS motor mapping: Comparing the absolute reliability of digital reconstruction methods to the golden standard. Brain Stimulation 12:309-313. https://doi.org/10.1016/j.brs.2018.11.005

Julkunen P (2014) Methods for estimating cortical motor representation size and location in navigated transcranial magnetic stimulation. Journal of Neuroscience Methods 232:125133. https://doi.org/10.1016/j.jneumeth.2014.05.020

Kallioniemi E, Julkunen P (2016) Alternative Stimulation Intensities for Mapping Cortical Motor Area with Navigated TMS. Brain Topography 29:395-404. https://doi.org/10.1007/s10548-016-0470-x 
Klochkov AS, Khizhnikova AE, Nazarova MA, Chernikova LA (2018) Pathological Upper Limb Synergies of Patients with Poststroke Hemiparesis. Neuroscience and Behavioral Physiology 48:. https://doi.org/10.1007/s11055-018-0634-0

Krieg SM, Lioumis P, Mäkelä JP, et al (2017) Protocol for motor and language mapping by navigated TMS in patients and healthy volunteers; workshop report. Acta Neurochirurgica 159:1187-1195. https://doi.org/10.1007/s00701-017-3187-z

Latash ML, Scholz JP, Schöner G (2007) Toward a new theory of motor synergies. Motor Control 11:276-308

Lefaucheur JP, Picht T (2016) The value of preoperative functional cortical mapping using navigated TMS. Neurophysiologie Clinique 46:125-133. https://doi.org/10.1016/j.neucli.2016.05.001

Leo A, Handjaras G, Bianchi M, et al (2016) A synergy-based hand control is encoded in human motor cortical areas. eLife 5:e13420. https://doi.org/10.7554/eLife.13420

Marconi B, Pecchioli C, Koch G, Caltagirone C (2007) Functional overlap between hand and forearm motor cortical representations during motor cognitive tasks. Clinical Neurophysiology 118:1767-1775. https://doi.org/10.1016/j.clinph.2007.04.028

Massé-Alarie H, Bergin MJG, Schneider C, et al (2017) "Discrete peaks" of excitability and map overlap reveal task-specific organization of primary motor cortex for control of human forearm muscles. Human Brain Mapping 38:6118-6132.

https://doi.org/10.1002/hbm.23816

Melgari J, Pasqualetti P, Pauri F, Rossini PM (2008) Muscles in “" Concert "': Study of Primary Motor Cortex Upper Limb Functional Topography. PLoS ONE 3:e3069. https://doi.org/10.1371/journal.pone.0003069

Nazarova M, Novikov P, Ivanina E, et al (2021) Mapping of multiple muscles with transcranial magnetic stimulation: absolute and relative test-retest reliability. Human Brain Mapping 42:1-21. https://doi.org/10.1002/hbm.25383

Nielsen JF (1996) Logarithmic distribution of amplitudes of compound muscle action potentials evoked by transcranial magnetic stimulation. Journal of Clinical Neurophysiology 13:423434. https://doi.org/10.1097/00004691-199609000-00005

Nieminen JO, Koponen LM, Ilmoniemi RJ (2015) Experimental Characterization of the Electric Field Distribution Induced by TMS Devices. Brain Stimulation 8:582-589. https://doi.org/10.1016/j.brs.2015.01.004

Nieminen JO, Koponen LM, Mäkelä N, et al (2019) Short-interval intracortical inhibition in human primary motor cortex: A multi-locus transcranial magnetic stimulation study. Neurolmage 203:116194. https://doi.org/10.1016/j.neuroimage.2019.116194

Nogueira-Campos AA, Oliveira LAS de, Della-Maggiore V, et al (2014) Corticospinal Excitability Preceding the Grasping of Emotion-Laden Stimuli. PLOS One 9:e94824. https://doi.org/10.1371/journal.pone.0094824

Novikov PA, Nazarova MA, Nikulin V v. (2018) TMSmap - Software for Quantitative Analysis of TMS Mapping Results. Frontiers in Human Neuroscience 12:239. https://doi.org/10.3389/fnhum.2018.00239 
Overduin SA, d'Avella A, Carmena JM, Bizzi E (2012) Microstimulation Activates a Handful of Muscle Synergies. Neuron 76:1071-1077. https://doi.org/10.1016/j.neuron.2012.10.018

Pearce A, Thickbroom GW, Byrnes ML, Mastaglia FL (2000) Functional Organisation of the Corticomotor Projection to the Hand in Skilled Functional reorganisation of the corticomotor projection to the hand in skilled racquet players. Experimental Brain Research 130:238-243. https://doi.org/10.1007/s002219900236

Pellegrini M, Zoghi M, Jaberzadeh S (2018) The effect of transcranial magnetic stimulation test intensity on the amplitude, variability and reliability of motor evoked potentials. Brain Research 1700:190-198. https://doi.org/10.1016/j.brainres.2018.09.002

Penfield W, Rasmussen T (1950) The Cerebral Cortex of Man. MacMillan New York

Perez MA, Rothwell JC (2015) Distinct influence of hand posture on cortical activity during human grasping. Journal of Neuroscience 35:4882-4889.

https://doi.org/10.1523/JNEUROSCI.4170-14.2015

Picht T, Frey D, Thieme S, et al (2016) Presurgical navigated TMS motor cortex mapping improves outcome in glioblastoma surgery: a controlled observational study. Journal of Neuro-Oncology 126:535-543. https://doi.org/10.1007/s11060-015-1993-9

Raffin E, Siebner HR (2019) Use-Dependent Plasticity in Human Primary Motor Hand Area : Synergistic Interplay Between Training and Immobilization. Cerebral Cortex 29:356-371. https://doi.org/10.1093/cercor/bhy226

Romero JR, Ramirez DM, Aglio LS, Gugino LD (2011) Brain mapping using transcranial magnetic stimulation. Neurosurgery Clinics of North America 22:141-152. https://doi.org/10.1016/j.nec.2010.11.002

Rubner Y, Tomasi C, Guibas L (1998) The Earth Mover's Distance as a Metric for Image Retrieval. International Journal of Computer Vision 40:99-121. https://doi.org/https://doi.org/10.1023/A:1026543900054

Schieber MH (2001) Constraints on somatotopic organization in the primary motor cortex. Journal of Neurophysiology 86:2125-2143. https://doi.org/10.1152/jn.2001.86.5.2125

Selvanayagam VS, Riek S, Carroll TJ (2012) A systematic method to quantify the presence of cross-talk in stimulus-evoked EMG responses: Implications for TMS studies. Journal of Applied Physiology 112:259-265. https://doi.org/10.1152/japplphysiol.00558.2011

Seynaeve L, Haeck T, Gramer M, et al (2019) Optimized preoperative motor cortex mapping in brain tumors using advanced processing of transcranial magnetic stimulation data. Neurolmage: Clinical 21:101657 Contents. https://doi.org/10.1016/j.nicl.2019.101657

Souza VH, Matsuda RH, Peres ASC, et al (2018) Development and characterization of the InVesalius Navigator software for navigated transcranial magnetic stimulation. Journal of Neuroscience Methods 309:109-120. https://doi.org/10.1016/j.jneumeth.2018.08.023

Souza VH, Vieira TM, Peres ASC, et al (2017) Effect of TMS coil orientation on the spatial distribution of motor evoked potentials in an intrinsic hand muscle. Biomedical Engineering / Biomedizinische Technik 63:635-645. https://doi.org/10.1515/bmt-20160240 
Strother L, Medendorp WP, Coros AM, Vilis T (2012) Double representation of the wrist and elbow in human motor cortex. European Journal of Neuroscience 36:3291-3298. https://doi.org/10.1111/j.1460-9568.2012.08241.x

Tharin S, Golby A (2007) Functional brain mapping and its applications to neurosurgery. Neurosurgery 60:185-202. https://doi.org/10.1227/01.NEU.0000255386.95464.52

Thordstein M, Saar K, Pegenius G, Elam M (2013) Individual effects of varying stimulation intensity and response criteria on area of activation for different muscles in humans. A study using navigated transcranial magnetic stimulation. Brain Stimulation 6:49-53. https://doi.org/10.1016/j.brs.2012.01.004

Tyc F, Boyadjian A (2005) Motor cortex plasticity induced by extensive training revealed by transcranial magnetic stimulation in human. European Journal of Neuroscience 21:259266. https://doi.org/10.1111/j.1460-9568.2004.03835.x

van de Ruit M, Grey MJ (2016) The TMS Map Scales with Increased Stimulation Intensity and Muscle Activation. Brain Topography 29:56-66. https://doi.org/10.1007/s10548-0150447-1

Van De Ruit M, Perenboom MJL, Grey MJ (2015) TMS brain mapping in less than two minutes. Brain Stimulation 8:231-239. https://doi.org/10.1016/j.brs.2014.10.020

Vishram S (2014) Textbook of Anatomy: Upper Limb and Thorax, 2nd edn. Reed Elsevier India Private Limited

Wassermann EM, McShane LM, Hallett M, Cohen LG (1992) Noninvasive mapping of muscle representations in human motor cortex. Electroencephalography and clinical neurophysiology 85:1-8. https://doi.org/10.1016/0168-5597(92)90094-R

Wilson SA, Thickbroom GW, Mastaglia FL (1993) Transcranial magnetic stimulation mapping of the motor cortex in normal subjects. Journal of the Neurological Sciences 118:134-144. https://doi.org/10.1016/0022-510X(93)90102-5

\section{Appendix 1}

The cortical motor maps were created in the TMSmap software (Novikov et al. 2018). The software uses the coil center coordinates to fit the closest spherical surface in the least square sense. It projects them to generate a surface in a region called the patch of interest, where a quasi-regular grid is constructed. Spatial filtering is applied to merge stimulation coordinates located closer than $3 \mathrm{~mm}$ to compensate for the inherent errors and fluctuations of the neuronavigation system and to avoid strong influence from outliers. The mean coordinates and the median peak-to-peak MEP amplitudes of the merged coordinates are projected on the grid, and interpolation with a smoothly changing function approach is applied to construct the map. The maximal radius of the stimulation site influence on the cortical surface was set to 15 $\mathrm{mm}$ according to the approximate full width at half maximum (FWHM) of the electric field distribution on the cortical surface (Nieminen et al. 2015).

The area, volume, and centroid were computed from the cortical motor maps. The map area and volume were calculated according to the equations below: 


$$
\begin{aligned}
\text { Area } & =\sum_{i=1}^{N} \Delta \mathrm{s}_{\mathrm{i}} \\
\text { Volume } & =\sum_{\mathrm{i}=1}^{\mathrm{N}} \mathrm{MEP}_{\mathrm{i}} \cdot \Delta \mathrm{s}_{\mathrm{i}}
\end{aligned}
$$

where $\mathrm{N}$ is the number of square grid elements with area $\Delta \mathrm{s}_{\mathrm{i}}$ and $\mathrm{MEP}_{\mathrm{i}}$ is the peak-to-peak amplitude. MEP amplitudes smaller than $50 \mu \mathrm{V}$ were discarded from the area and volume estimates (Groppa et al. 2012). The map area represents the extent of the cortical motor representation, and the volume represents the map area weighted by the MEP amplitude. The volume is better described as an effective area. Considering a map within a particular area, the height of the map is the MEP amplitude, and it represents how strong the muscle's response was to the stimuli applied in that area. Comparing two maps with the same area and different volumes, the extent of that muscle's representation on M1 is equal, although the map whose height is higher has stronger muscle recruitment and representation. The centroid was calculated as:

$$
\text { centroid }=\left(\frac{\sum_{\mathrm{i}=1}^{\mathrm{N}}\left(\mathrm{h}_{\mathrm{i}} * \mathrm{~s}_{\mathrm{i}} * \mathrm{x}_{\mathrm{i}}\right)}{\sum_{\mathrm{i}=1}^{\mathrm{N}}\left(\mathrm{h}_{\mathrm{i}} * \mathrm{~s}_{\mathrm{i}}\right)}, \frac{\sum_{\mathrm{i}=1}^{\mathrm{N}}\left(\mathrm{h}_{\mathrm{i}} * \mathrm{~s}_{\mathrm{i}} * \mathrm{y}_{\mathrm{i}}\right)}{\sum_{\mathrm{i}=1}^{\mathrm{N}}\left(\mathrm{h}_{\mathrm{i}} * \mathrm{~s}_{\mathrm{i}}\right)}, \frac{\sum_{\mathrm{i}=1}^{\mathrm{N}}\left(\mathrm{h}_{\mathrm{i}} * \mathrm{~s}_{\mathrm{i}} * \mathrm{z}_{\mathrm{i}}\right)}{\sum_{\mathrm{i}=1}^{\mathrm{N}}\left(\mathrm{h}_{\mathrm{i}} * \mathrm{~s}_{\mathrm{i}}\right)}\right)
$$

where $\mathrm{N}$ is the number of grid elements, $\mathrm{h}_{\mathrm{i}}$ is the height of the constructed map above the grid element, $\mathrm{s}_{\mathrm{i}}$ is the area and $\mathrm{x}_{\mathrm{i}}, \mathrm{y}_{\mathrm{i}}$, and $\mathrm{z}_{\mathrm{i}}$ are the coordinates of the center of the grid element (Novikov et al. 2018).

The overlap map was constructed considering the area where MEP amplitudes were greater than $50 \mu \mathrm{V}$ for all the recorded muscles. The map height is the smallest MEP amplitude across all muscles at each grid element. The area, volume, centroid and EMD for the overlap maps were calculated as described above. The size and topography parameters selected to assess the coactivation between adjacent muscles were area and volume OD, and EMD and centroids Euclidean distance, respectively, as described in Methods. 